\title{
A Fractal Origin for the Mass Spectrum of Interstellar Clouds: II. Cloud Models and Power Law Slopes
}

\author{
Bruce G. Elmegreen \\ IBM Research Division, T.J. Watson Research Center, P.O. Box 218, Yorktown Heights, \\ NY 10598, USA
}

\begin{abstract}
Three-dimensional fractal models on grids of $\sim 200^{3}$ pixels are generated from the inverse Fourier transform of noise with a power law cutoff, exponentiated to give a log normal distribution of density. The fractals are clipped at various intensity levels and the mass and size distribution functions of the clipped peaks and their subpeaks are determined. These distribution functions are analogous to the cloud mass functions determined from maps of the fractal interstellar medium using various thresholds for the definition of a cloud. The model mass functions are found to be power laws with powers ranging from -1.6 to -2.4 in linear mass intervals as the clipping level increases from $\sim 0.03$ to $\sim 0.3$ of the peak intensity. The low clipping value gives a cloud filling factor of $\sim 10 \%$ and should be a good model for molecular cloud surveys. The agreement between the mass spectrum of this model and the observed cloud and clump mass spectra suggests that a pervasively fractal interstellar medium can be interpreted as a cloud/intercloud medium if the peaks of the fractal intensity distribution are taken to be clouds. Their mass function is a power law even though the density distribution function in the gas is a log-normal. This is because the size distribution function of the clipped clouds is a power law, and with clipping, each cloud has about the same average density. A similar result would apply to projected clouds that are clipped fractals, giving nearly constant column densities for power law mass functions. The steepening of the mass function for higher clip values suggests a partial explanation for the steeper slope of the mass functions for star clusters and $\mathrm{OB}$ associations, which sample denser regions of interstellar gas. The mass function of the highest peaks is similar to the Salpeter IMF, suggesting again that stellar masses may be determined in part by the geometry of turbulent gas.
\end{abstract}

Subject headings: turbulence - ISM: clouds - ISM: structure — open clusters and associations: general 


\section{Introduction}

Interstellar gas appears scale-free when viewed with Fourier transform power spectra (Crovisier \& Dickey 1983; Green 1993; Lazarian \& Pogosyan 2000; Stützki et al. 1998; Stanimirovic et al. 1999; Elmegreen, Kim, \& Staveley-Smith 2001), delta variance techniques (Stützki et al. 1998; Zielinsky \& Stützki 1999), spectral correlation functions (Rosolowsky et al. 1999), principal component analysis (Heyer \& Schloerb 1997), perimeter-area measures (Dickman, Horvath, \& Margulis 1990; Falgarone, Phillips, \& Walker 1991), box-counting techniques (Westpfahl et al. 1999), and multifractal analysis (Chappell \& Scalo 2001).

The interstellar medium (ISM) looks like a collection of discrete clouds, however, when intensity contours are drawn (Solomon et al. 1987; Loren 1989; Stützki \& Güsten 1990; Williams, de Geus, \& Blitz 1994; Lemme et al. 1995; Kramer et al. 1998; Heyer, Carpenter, \& Snell 2001) or when spectral absorption lines are fit to Gaussians (Adams 1949; Hobbs 1978; Clark 1965; Radhakrishnan \& Goss 1972; Spitzer \& Jenkins 1975).

These two interpretations have led to distinct models for the origin of gas structure and star formation. Scale-free models typically involve turbulence and self-gravity (Falgarone \& Phillips 1990; Scalo 1990; Pfenniger \& Combes 1994; Lazarian 1995; Elmegreen 1999b; Rosolowsky et al. 1999; MacLow \& Ossenkopf 2000; Pichardo et al. 2000; Klessen, Heitsch, \& MacLow 2000; Vázquez-Semadeni, Gazol, \& Scalo 2000; Semelin \& Combes 2000; Ostriker, Stone, \& Gammie 2001; Heitsch, Mac Low, \& Klessen 2001; Toomre \& Kalnajs 1991; Wada \& Norman 1999, 2001).

Cloudy models involve sticky collisions and star formation triggered by colliding and compressed clouds (Kwan 1979; Hunter et al. 1986; Tan 2000; Scoville, Sanders, \& Clemens 1986).

For general interstellar gas dynamics, the turbulent model (von Weizsacker 1951; Sasao 1973) may be more realistic than the cloudy model for the origin of structure (LaRosa, Shore \& Magnani 1999; Ballesteros-Paredes, Hartmann, \& Vázquez-Semadeni 1999). Turbulence also gives cloud-like spectral lines through both density structure and velocity crowding (Ballesteros-Paredes, Vázquez-Semadeni, \& Scalo 1999; Lazarian \& Pogosyan 2000; Pichardo, et al. 2000).

For sudden transition fronts like expanding shells, spiral arms, and dust lanes, cloud collisions may be an appropriate way to model the dynamics (e.g., Kenney \& Lord 1991; Elmegreen 1988). This is because the pre-front clumps made by turbulence at slow ambient speeds are forced to collide together and interact at much faster speeds inside the front. Strongly self-gravitating clouds that are made by turbulence at moderate speeds but then given a chance to cool and settle to high densities should also interact as in the cloudy models. 
Because of their high densities, these clouds or clumps should move somewhat independently of the surrounding turbulent gas, perhaps lagging behind the expanding flows to make bright rims, or punching through spiral dustlanes to make feathery structures (Seth 2000).

The most obvious point of contact between these two views of gas structure is the mass spectrum of the regions that are isolated enough to be defined as clouds. The scale-free nature of the gas shows up as a scale-free mass spectrum for the clouds (Elmegreen \& Falgarone 1996; Stützki et al. 1997). This mass spectrum is not useful in the turbulent model because it does not reflect the continuous distribution of matter that is really present. The spectrum is more important for bound star clusters, which are better defined (Elmegreen et al. 2000), and for individual stars, whose masses are probably proportional to the primordial clump masses (Motte, André, \& Neri 1998; Testi \& Sargent 1998; Bacmann et al. 2000; Tachihara et al. 2000).

Here we model the cloud mass and size spectra using the intensity peaks in a simulated fractal to represent clouds. The results show the anticipated $\sim M^{-2} d M$ spectrum that comes from simple hierarchical models (Fleck 1996), but for a different reason than what is usually given. In the usual interpretation, the $M^{-2} d M$ spectrum comes from the fact that there is a constant total mass in each logarithmic interval of mass; i.e., each small clump at the bottom is contained inside each large clump at the top. Then $M \xi(\log M) d \log M=$ constant $\times d \log M$ becomes $n(M) d M \equiv \xi(\log M) d \log M=\xi(\log M) d M / M \propto M^{-2} d M$. We show here that the bottom of the hierarchy, where the gas density has local isolated peaks, has about the same spectrum. This is true even though the probability distribution function of density alone is not a power law, but log-normal.

We also find that the spectrum flattens to $M^{-1.6} d M$ as the lower limit to the cloud intensity is decreased. This flattening may explain the difference between the mass spectrum of star clusters, which is close to $M^{-2} d M$ (Battinelli et al. 1994; Elmegreen \& Efremov 1997; Whitmore \& Schweizer 1995; Zhang \& Fall 1999) and the mass spectrum for clouds, which typically ranges between $M^{-1.5}$ and $M^{-1.8}$ (e.g., Blitz 1993; Kramer et al. 1998; Heyer, Carpenter, \& Snell 2001). An even steeper spectrum results from the highest clipping levels modeled here and is close to the Salpeter IMF for stars. Thus the increase in slope of the mass functions from clouds to clusters to stars may be partly the result of different density thresholds in the same fractal gas. 


\section{Models}

Fractal Brownian motion clouds (Stutzki et al. 1998) are generated by first filling a 3D lattice in wavenumber space, $\left(k_{x}, k_{y}, k_{z}\right)$, with noise distributed as a Gaussian with

a dispersion of unity. The noise cube is multiplied by $k^{-5 / 3}$ for $k=\left(k_{x}^{2}+k_{y}^{2}+k_{z}^{2}\right)^{1 / 2}$. The inverse three-dimensional FFT of the resulting truncated noise cube gives a fractal (Voss 1988) with a Gaussian distribution of intensity, $I_{0}(x, y, z)$, as shown on the bottom of Figure 1. To simulate a turbulent fractal, we exponentiate this intensity distribution, $I(x, y, z)=\exp \left(\alpha I_{0}(x, y, z) / I_{0, \max }\right)$ for maximum original intensity $I_{0, \text { max }}$ and contrast factor $\alpha=4$. This gives another fractal, now with a log-normal density distribution (Fig. 1, top). The motivation for the log-normal comes from studies by Vázquez-Semadeni (1994), Nordlund \& Padoan (1999), Klessen 2000, and Wada \& Norman (2001).

A fractal obtained in this way is a continuous distribution of density and more properly called a multifractal because the local fractal dimension of the density structure varies from the peaks to the valleys (e.g., Chappell \& Scalo 2001; Vavrek 2001). We refer to it here only as a fractal.

For the cloud mass and size spectra determined here, we consider two cloud models. In the first (Sect. 2.1), a cloud is defined as all of the emission above a fixed cutoff in density. In the second (Sect. 2.2), clouds that are resolved as separate peaks are each counted, whether or not they occur inside a broader emission region above the cutoff. The broader emission in this second case is divided into subparts to go with each peak. The subpart masses are taken proportional to the peak masses that were previously determined from a higher cutoff level. The algorithm will be described in more detail later.

For the first set of models, the fractal density distribution in which the clouds were counted was taken to be the inner $180^{3}$ pixels of a $210^{3}$ grid, to avoid edge effects. In the second case, the inner $160^{3}$ px inside a $192^{3}$ px grid was used. Models with many different grid sizes were made to confirm that the basic results are independent of this. Larger grids have more clouds; the first model had 73,000 separate clouds above 0.03 times the peak density, and the second model had 40,000 clouds and subclouds above $e^{-3}=0.05$ times the peak density. The ratio of peak to minimum value in the density distribution was 2400 in the first set of models, and 3800 in the second set; this ratio is determined by the $\alpha$ value.

\subsection{Model 1: Clouds as Emission Regions above a Fixed Density Cutoff}

The central $100^{3}$ pixels of the first model is shown in Figure 2, clipped to display everything brighter than 0.03 of the peak. This clipping level gives the most reasonable 
agreement with interstellar cloud surveys, as discussed below.

To find clouds in the $180^{3} \mathrm{px}^{3}$ fractal, we begin by initiating another $180^{3}$ cube, $J(x, y, z)$, with values all equal to 0 . This cube keeps track of which pixels in the fractal cube have been counted in the mass sum. Then, to find a cloud, we begin 10 pixels inside each edge in one corner of the fractal cube and step along in one direction in a regular fashion until a pixel value is reached that exceeds the clipping threshold. We determine whether this pixel has been counted before by viewing the value of the corresponding pixel in $J(x, y, z)$. If $J=0$, then it has not been counted before and we proceed to count the cloud mass. If $J=1$, we continue with our regular search for another cloud. In the case where $J=0$ for this pixel, we add the density of the pixel to the total mass of this cloud, which begins at 0 , and we change the corresponding pixel in the $J$ cube from 0 to 1 . We then begin to map around inside this cloud by stepping one pixel away from the current pixel in a random direction. If the new pixel has a value lower than the threshold, we have just crossed the cloud boundary so we go back. If the new pixel value is greater than the threshold so we are still inside the cloud, then we determine if this position has already been added to the cloud mass sum by reading the value of $J$ at this position. If the new pixel has not been counted in the current cloud (i.e., if the $J$ value is still 0 ), then we add its density to the running sum for that cloud and set the corresponding value in $J$ to 1 .

This procedure continues until further random searching returns no new pixels in the same cloud after a total number of additional tries equal to $4 N^{2}$, where $N$ is the number of pixels already counted for that cloud. If $N$ is small or large, then the minimum or maximum trial counts were taken equal to 1000 and $10^{7}$. This step number requirement gives the random walk among pixels a reasonable chance to visit every pixel inside the cloud, even if the boundary is very ragged and there are cul de sacs and spikes which would nearly trap a shorter random search. When the cloud mass is summed, we add the result to a histogram of cloud masses, which is the mass spectrum, using a linear interval of the mass for counting. The cloud search then continues along the regular search path where it was before the most recent cloud was mapped. Cloud searches are much faster for higher intensity cut-off levels because the clouds are smaller and the search requirement of $4 N^{2}$ steps without a new cloud pixel is more easily reached.

The clouds modeled here are solid objects, not projected objects. We consider cloud structure in the full three-dimensional grid because spectral line mapping in real data can usually distinguish between different three-dimensional objects at different velocities on the same line of sight. Thus the fractal dimension of observed interstellar CO clouds and clumps, obtained from the size distribution of contour-mapped objects, was found to be around 2.3 one more than the projected fractal dimension of 1.3 obtained from the boundary structure 
(Pfenniger \& Combes 1994; Elmegreen \& Falgarone 1997). This approximation does not account for artificial clouds that may appear in real surveys because of velocity crowding in turbulent gas (Lazarian \& Pogosyan 2000).

Figure 3 shows mass spectra for intensity clipping values equal to $0.03,0.1$, and 0.3 times the peak intensity. The spectrum for the 0.3 clipping level is an average of the spectra for 10 different random fractal models; this averaging was done in order to reduce the noise in the cloud counting, considering the small number of bright peaks in each model. The spectrum for the 0.1 clipping level is an average over 4 random fractal models, and the spectrum for 0.03 is an average over 2 fractal models. The spectral slopes are approximately $\alpha=-1.7,-1.9$ and -2.3 , respectively. These correspond to power law mass functions $n(M) d M \propto M^{\alpha} d M$ in linear intervals of mass, or mass functions $\propto M^{1+\alpha} d \log M$ in logarithmic intervals of mass.

Figure 4 shows the running average slopes of the mass functions versus the cloud mass for different clipping levels. These average slopes start at the lowest cloud mass for that clipping level and extend up to the mass $M$ plotted on the abscissa. The slopes of the power law mass spectra become inaccurate at high mass because the number of clouds goes to zero and the individual spectra level off. At low to intermediate mass, the slopes are about constant for a range in cloud mass that spans a factor of $\sim 100$ for low clipping levels. This is the mass range where the spectrum is a power law. The range is smaller for higher clipping levels because the cloud masses are smaller.

The slope of the power law mass spectrum gets steeper with higher clipping levels. To understand the origin of this increase, we ran other models with smaller peak-to-valley density contrasts (e.g., using $\alpha=1.4$ ). The same range in clump spectral slopes resulted for the high and low clipping levels, but now the range in clipping levels was only a factor of 5 because of the smaller density contrast for the low $\alpha$ case, rather than 30 as in the Figures. This change suggests that the mass spectrum slope decreases for low clipping values in both cases because the low density limit of the fractal is approached. Near the low density limit, each cloud has a sprawling boundary that spans nearly the distance to the next cloud, and there is a higher proportion of high mass clouds. There is also very little intercloud medium at low clipping levels. For the lowest clipping level, 0.03 times the peak, the threshold cloud density is $\sim 70$ times the minimum fractal density, which is $\sim 1 / 2400$ of the peak. This is still far from the lowest density level, but apparently close enough to change the geometric characteristics.

Figure 5 shows the size distribution function for the model clipped at 0.03 times the peak value. The cloud size is taken to be the cube-root of the number of pixels. The size distribution function is approximately a power law with a slope of $\sim-3.75$. This plot is again a histogram, determined for linear intervals of size. Thus the average fractal dimension 
is one more than this slope, or $D \sim-2.75$ (Mandelbrot 1982). This is not a very meaningful concept for a multifractal with a smooth distribution of density because there is a range of fractal dimensions depending on location, but the result is analogous to the dimension determined from the size distribution of interstellar clouds, which gives about $D \sim-2.3$ (Elmegreen \& Falgarone 1996). The size spectra for the models with other clipping factors are not shown because the size ranges are too small to be interesting; they tend to be steeper for higher clipping levels like the mass spectra.

Figure 6 shows the mass versus size for all clouds. The regressions with slopes of $\sim 3.3$ indicate that all clouds found with a particular clipping level have about the same average density and that the density scales in proportion to the clipping level. This is to be expected because most of the cloud mass is on the periphery of the cloud, where the volume is greatest, and the density there is about the clipping value. This cubic dependence is similar to that found for clump studies inside molecular clouds (Loren 1989; Stützki \& Güsten 1990; Williams, de Geus, \& Blitz 1994; Williams, Blitz, \& Stark 1995; see the summary of observations in Elmegreen \& Falgarone 1996) and differs significantly from the quadratic dependence found by Larson (1981).

The difference in the mass-radius relations for clump surveys and whole GMC surveys seems to be related to the threshold used for the observation. In the clump case, the threshold is probably the critical density for excitation of the molecule (as recognized, for example, by Williams, Blitz, \& Stark 1995). In Larson's correlation, which was based on large scale CO surveys and later confirmed by other whole-cloud surveys (e.g., Solomon et al. 1987), the threshold is CO column density, probably tied to the telescope sensitivity. For example, the Solomon et al. survey used a brightness temperature limit to define CO clouds. Thus the fundamental $M \propto R^{2}$ result of Larson (1981), which, when combined with the virial theorem and Kolmogorov law, is the basis for most of the correlations that have been found for molecular clouds, seems to be an artifact of sampling near the telescope limit of detection, which is a column density limit for a fractal cloud (see also Vázquez-Semadeni, BallesterosParedes, \& Rodriguez, 1997).

\subsection{Model 2: Clouds and Subclouds Defined by Each Peak}

In the second model, clouds were defined in a $160^{3} \mathrm{px}^{3}$ grid by local peaks in the density, whether or not they were separated by a region below the current density cutoff. This is analogous to the definition of a cloud or clump in the surveys by Williams et al. (1994, 1995) and Stützki, \& Güsten (1990), who fit the peaks and their surrounding emission with various strategies. This model differs from the first model discussed above because a single 
cloud in the first case could be divided into several sub-clouds in the second case if there are separate peaks. To keep track of where the peaks are, we used a third cube, $P(x, y, z)$, of size $160^{3}$ with entries equal to the peak number. A second cube, $J(x, y, z)$ as in model 1 , kept track of which pixels had been searched already.

The peaks in the density are found by successively lowering the threshold, which begins at $\exp (-1)$ times the overall peak density in the fractal cube, and then goes to $\exp (-2)$ and $\exp (-3)$ times this peak density. The peaks found with the $\exp (-1)$ threshold are located in the same way as for model 1, with no separate subpeaks. Presumably they would be unresolved also in a real cloud if the peak intensity stands above the detection threshold by a factor less than $e^{1}=2.7$. These first-threshold clouds are then used by the program again, but now with the threshold of $\exp (-2)$. When a new cloud was found standing above this $\exp (-2)$ threshold, the peak-identification cube, $P$, was viewed at all of the positions belonging to this new cloud to see if there were already any peaks in it. If there were no peaks, then the current cloud was defined to be a new peak and its full mass was added to the list of peaks. If there was one previous peak, then the mass from this level was given to the previous peak. If there was more than one previous peak from the next higher cutoff level, then the mass of the current cloud was divided up among the higher peaks. With this strategy, a distinct peak is a region separated from another peak by a level of emission that dips below $\sim \exp (-1)$ of the peak value.

This partitioning of mass among the various peaks in a threshold-defined cloud was done in several different ways. In one method, the peaks were fit to Gaussians when they were first found, and the extrapolated masses from these Gaussians were determined at the next lower level when they appeared inside a cloud there. This mass at the next lower level was then divided up among the former peaks in proportion to their extrapolated Gaussian masses. In another method, the current cloud was divided into pieces proportional to the former peak masses themselves, without any Gaussian extrapolations. The difference between these two partitionings was noticeable but not large. Because the peak masses from the levels where they are first discovered are distributed as a power law, as shown in the previous subsection, any partitioning of the current cloud in proportion to these previous peaks maintains that same power law and extends it to larger masses. Thus the combined mass distribution of isolated peaks and subdivided clouds is similar to that of the peaks alone. In what follows, we discuss this case, i.e., where the partitioning is proportional to the previous peak masses. In the Gaussian-fitting case, the extrapolated Gaussian masses could be much larger than the pure peak masses if the Gaussian dispersions were large, and this led to a wild variation in how the clouds in the current level were divided among peaks from the higher levels. It gave results that were similar to a third case where the current cloud was equally divided among the peaks from the higher level. 
In the final spectrum down to the third level $\left(e^{-3}\right)$, the Gaussian and equal-division methods did not produce a continuous power law, but had a power law at low mass, where the peaks were single, and a broad concentration of masses at intermediate values, where the subdivisions gave a somewhat random mixture of masses. The preferred case, with the subdivisions directly proportional to the former peak masses, gave an approximately continuous power law.

Figure 7 shows the mass distribution functions for clouds and sub-clouds defined in this way using density cutoffs of $\exp (-1)$ (on the left), $\exp (-2)$, and $\exp (-3)$ (on the right). The top three panels have no added noise, the bottom three have noise at the level of $0.5 e^{-3}$ times the peak density. This noise affects only the mass spectrum made from the lowest contour (plotted on the right), and it tends to add low mass clouds, which are mostly noise. The average slope of the mass spectrum does not change much with added noise.

The dashed lines that lie nearly parallel to the distribution functions in Figure 7 have slopes of $-2.4,-1.8$, and -1.6 for these three cutoffs, respectively. These slopes are similar to the mass spectrum slopes found in the previous section for the cases with similar density cutoffs. Thus the division of big clouds into subclouds does not affect the spectrum much. There is a tendency for the mass spectra to become slightly steeper at low mass, which is the result of the steepening effect found previously for small clouds at high cutoff levels: most of these low mass clouds are single peaks near the threshold. Figure 8 shows the running average slopes for the noise-free cloud/subcloud case, as in Figure 4. The other figures shown in section 2.1, namely, the mass-size relation and the size spectrum, are essentially the same for the present case.

\section{Discussion}

The connection between the cloud model of interstellar structure and the multifractal model is readily understood when clouds are viewed as isolated peaks in the fractal. Here we have shown that the mass spectrum for such fractal clouds is similar to the observed cloud spectrum when clouds are defined or selected to be those regions where the local density exceeds several percent of the peak (resolved) density, or when clouds are defined to be the resolvable peaks plus a proportional amount of gas in the underlying plateaus. Unresolved peaks can have higher densities, as can a fractal model with more cells, but this will not affect the mass spectrum of the resolved objects.

Observational cloud surveys tend to span only a factor of 5 to 10 in physical scale (see references for cloud and clump surveys given above). This limited range is entirely a 
selection effect because unbiased surveys analyzed with power spectra or similar techniques find a much wider range of scales in the interstellar medium, up to a factor greater than $\sim 100$ in the case of whole galaxies. In the cloud surveys, the factor of $\sim 10$ limit arises because smaller regions are unresolved and larger regions are subdivided into separate clouds, the whole structure being hierarchical (Scalo 1985).

For many $\mathrm{CO}$ surveys, a factor of 10 in scale corresponds to a factor of 10 in average density (Larson 1981), and a factor of 10 in density corresponds to a volume filling factor of $10 \%$. This latter result is because for an infinitely self-similar fractal, the filling factor scales inversely with the density (Elmegreen 1999c). In fact, most cloud surveys report a filling factor of about $\sim 10 \%$ (e.g., see review in Blitz 1993); this is only an artifact of sampling in the fractal point of view. In the current models, the average filling factors for the clouds in Section 2.1 are $1.2 \times 10^{-4}, 7.0 \times 10^{-3}$, and 0.13 for clipping densities of $0.3,0.1$, and 0.03 times the peak. For the clouds in section 2.2, they are $1.3 \times 10^{-4}, 5.7 \times 10^{-3}$, and 0.065 .

These filling factors vary approximately as the inverse cube or inverse square of the density threshold because the low clipping levels approach the minimum density, and the sprawling cloud boundaries take up excess volume. The models with the lowest clipping levels, 0.03 and $e^{-3}=0.05$, have the about same filling factors as the observed clouds and clumps in surveys.

The point here is that the density range that is inadvertently selected for real cloud surveys is also what the fractal models need to give the observed filling factor and mass function. If different observing techniques are employed, giving a wider range of cloud sizes or average densities, for example, thereby increasing the clump filling factor, then we predict that the slope of the resulting mass function will decrease. Similarly, surveys with molecular tracers sensitive to very high densities should be selecting regions closer to the peaks of the turbulent fractal, with lower filling factors, and these surveys should get slightly steeper slopes for the clump mass spectrum.

Note that the absolute density of an observed region is not important for the slope of the mass spectrum, only the relative density compared to the peaks and valleys in that region. As the threshold density approaches the minimum density, the mass spectrum flattens because the cloud boundaries spread out and the low density "intercloud" medium, which is just the sub-threshold gas in the same fractal, gets included more and more with each cloud. This interpretation is valid for molecular clouds and other gases with a single phase of thermal temperature. Of course, the intercloud means something different when there is a high temperature phase. For example, fractal models of the neutral hydrogen in the Large Magellanic Cloud require a multi-phase structure because of the high density contrasts that are present (Elmegreen, Kim, \& Staveley-Smith 2001). 
The size distribution function for our model clouds is also similar to that for interstellar clouds in the best-fit case. The power law nature of the size spectrum, combined with the cutoff in density in the definition of a cloud, ensures that the mass spectrum is also a power law. This is because the average density is always some factor of order unity times the cutoff. Thus the mass spectrum is a power law even though the density distribution function is a log-normal.

The mass spectrum for star clusters is steeper than that for clumps, by several tenths (cf. Sect. 1). The fractal model suggests that this steepness results from the denser cloud regions that are sampled by clusters. The typical density of an embedded star cluster corresponds to an $\mathrm{H}_{2}$ density of around $10^{5} \mathrm{~cm}^{-3}$ (Lada, Evans \& Falgarone 1997). This is much closer to the peak density of the turbulent fractal than the density threshold for the cloud itself, so the mass spectrum should be steeper in this model. However, gravity modifies the gas density in the region of a cluster, so the present results are only suggestive.

The densest peaks sampled here, with $>30 \%$ of the peak density in the cloud, have mass spectra with slopes of $\sim-2.3$ and $\sim-2.4$ for models 1 and 2 , respectively. These are similar to the slope of the Salpeter stellar IMF. Indeed, mm-continuum sources and stars do sample denser regions than clusters, so the increase in slope is sensible. However, other factors enter into the stellar IMF, such as the relative rate of collapse in regions with different masses and the competition for mass. Thus the importance of the purely fractal result for the IMF cannot be assessed without further modeling (see Elmegreen 1997, 1999a).

\section{Summary}

Clouds that are defined by the peaks of a smooth fractal distribution, whether deliberately or inadvertently because of the observing procedure, have power law mass distributions even if the gas density has a log-normal distribution. The power of the mass distribution ranges from -1.6 to -2.4 as the clipping level varies from 0.03 to 0.3 times the peak. The shallower power is similar to what is observed for interstellar clouds, and the cloud filling factor in the model is similar to the observations too. The steeper power laws are similar to what are observed for star clusters and mm-continuum sources, respectively, perhaps because clusters and continuum sources sample greater densities than the outer cloud boundary.

The origin of the power law for the cloud mass spectrum is the power law for the cloud size spectrum at a fixed threshold in density or column density, depending on the nature of the survey. Many of the correlations observed for molecular clouds and clumps now appear to be artifacts of these density and column density thresholds, considering the fractal nature 
of the interstellar medium and the internal correlations from turbulent motions.

Acknowledgements: This work was supported by NSF Grant AST-9870112 to B.G.E. Helpful comments by the referee led to the second clump-finding algorithm discussed here.

\section{REFERENCES}

Adams, W.S. 1949, ApJ, 109, 354

Bacmann, A., André, P., Puget, J.-L., Abergel, A., Bontemps, S., \& Ward-Thompson, D. 2000, in From Darkness to Light: Origin and Early Evolution of Young Stellar Clusters, eds. T. Montmerle \& P. André, ASP Conf. Series, in press

Ballesteros-Parades, J., Hartmann, L., \& Vázquez-Semadeni, E. 1999, ApJ, 527, 285

Ballesteros-Paredes, J., Vázquez-Semadeni, E., \& Scalo, J. 1999, ApJ, 515, 286

Battinelli P., Brandimarti A., \& Capuzzo-Dolcetta R. 1994, A\&AS, 104, 379

Blitz, L. 1993, in Protostars and Planets III, ed. E.H. Levy \& J.I. Lunine, Tucson: Univ. Arizona Press, p. 125

Chappell, D., \& Scalo, J. 2001, ApJ, 551, 712

Clark, B.G. 1965, ApJ, 142, 1398

Crovisier, J., \& Dickey, J.M. 1983, A\&A, 122, 282

Dickman, R. L., Horvath, M. A., \& Margulis, M. 1990, ApJ, 365, 586

Elmegreen, B.G. 1988, ApJ, 326, 616

Elmegreen, B.G. 1997, ApJ, 486, 944

Elmegreen, B.G. 1999a, ApJ, 515, 323

Elmegreen, B.G. 1999b, ApJ, 527, 277

Elmegreen, B.G. 1999c, in The Physics and Chemistry of the Interstellar Medium, Proceedings of the 3rd Cologne-Zermatt Symposium, eds. V. Ossenkopf, J. Stutzki, \& G. Winnewisser, Aachen: Shaker-Verlag, p. 77

Elmegreen, B.G., \& Falgarone, E. 1996, ApJ, 471, 816 
Elmegreen, B.G., \& Efremov, Yu. N. 1997, ApJ, 480, 235

Elmegreen, B.G., Efremov, Y.N., Pudritz, R., \& Zinnecker, H. 2000, in Protostars and Planets IV, eds. V. G. Mannings, A. P. Boss, \& S. S. Russell, Tucson: Univ. Arizona Press, p. 179

Elmegreen, B.G., Kim, S., \& Staveley-Smith, L. 2001, ApJ, 548, 749

Falgarone, E., \& Phillips, T. 1990, ApJ, 359, 399

Falgarone, E., Phillips, T. G., \& Walker, C. K. 1991, ApJ, 378, 186

Fleck, R.C., Jr. 1996, ApJ, 458, 739

Green, D.A. 1993, MNRAS, 262, 327

Heyer, M.H., \& Schloerb, F. P. 1997, ApJ, 475, 173

Heyer, M.H., Carpenter, J.M., \& Snell, R.L. 2001, ApJ, 551, 852

Heitsch, F., Mac Low, M.-M., \& Klessen, R.S. 2001, ApJ, 547, 280

Hobbs, L.M. 1978, ApJS, 38, 129

Hunter, J. H., Jr., Sandford, M. T., II, Whitaker, R. W., \& Klein, R. I. 1986, ApJ, 305, 309

Kenney, J.D.P., \& Lord, S.D. 1991, ApJ, 381, 118

Klessen, R.S. 2000, ApJ, 535, 869

Klessen, R.S., Heitsch, F., \& MacLow, M.-M. 2000, ApJ, 535, 887

Kwan, J. 1979, ApJ, 229, 567

Kramer, C., Stutzki, J., Rohrig, R., \& Corneliussen, U. 1998, A\&A, 329, 249

Lada, E.A., Evans, N.J., II., \& Falgarone, E. 1997, ApJ, 488, 286

LaRosa, T.N., Shore, S.N., \& Magnani, L. 1999, ApJ, 512, 761

Larson, R.B. 1981, MNRAS, 194, 809

Lazarian, A. 1995, A\&A, 293, 507

Lazarian, A., \& Pogosyan, D. 2000, ApJ, 537, 720

Lemme, C., Walmsley, C.M., Wilson, T.L., \& Muders, D. 1995, A\&A, 302, 509 
Loren, R.B. 1989, ApJ, 338, 902

MacLow, M.-M., \& Ossenkopf, V. 2000, A\&A, 353, 339

Mandelbrot, B.B. 1982, The Fractal Geometry of Nature, (San Francisco: Freeman).

Motte, F., André, P., \& Neri, R. 1998, A\&A, 336, 150

Nordlund, A., \& Padoan, P. 1999, in Interstellar Turbulence, eds. J. Franco \& A. Carramiñana, Cambridge: Cambridge University Press, p. 218

Ostriker, E.C., Stone, J.M., \& Gammie, C.F. 2001, ApJ, 546, 980

Pfenniger, D. \& Combes, F. 1994, A\&A, 285, 94

Pichardo, B., Vázquez-Semadeni, E., Gazol, A., Passot, T., Ballesteros-Paredes, J. 2000, ApJ, 532, 353

Radhakrishnan, V., \& Goss, W.M. 1972, ApJS, 24, 161

Rosolowsky, E.W., Goodman, A.A., Wilner, D.J., \& Williams, J.P. 1999, ApJ, 524, 887

Sasao, T. 1973, PASJ, 25, 1

Scalo, J.M., 1985, in Protostars and Planets II, ed. D.C. Black and M.S. Matthews, Tucson: University of Arizona, 201

Scalo, J. 1990, in Physical Processes in Fragmentation and Star Formation, eds. R. CapuzzoDolcetta, C. Chiosi, \& A. Di Fazio, Dordrecht: Kluwer, p. 151

Scoville, N. Z., Sanders, D. B., \& Clemens, D. P. 1986, ApJ, 310, L77

Semelin, B., Combes, F. 2000, 360, 1096

Seth, K. 2000, PhD. Dissertation, Univ. of Maryland

Solomon, P. M., Rivolo, A. R., Barrett, J., \& Yahil, A. 1987, ApJ, 319, 730

Spitzer, L., Jr., \& Jenkins, E.B. 1975, ARA\&A, 13, 133

Stanimirovic, S., Staveley-Smith, L., Dickey, J.M., Sault, R.J., \& Snowden, S.L. 1999, MNRAS, 302, 417

Stützki, J., Bensch, F., Heithausen, A., Ossenkopf, V., \& Zielinsky, M. 1998, A\&A, 336, 697

Stützki, J., \& Güsten, R. 1990, ApJ, 356, 513 
Tachihara, K., Hara, A., Obayashi, A., Yonekura, Y., Onishi, T., Mizuno, A., \& Fukui, Y. 2000, in From Darkness to Light: Origin and Early Evolution of Young Stellar Clusters, eds. T. Montmerle \& P. André, ASP Conf. Series, in press

Tan, J.C. 2000, ApJ, 536, 173

Testi, L., \& Sargent, A.I. 1998, ApJ, 508, L91

Toomre, A., \& Kalnajs, A.J. 1991, in Dynamics of Disk Galaxies, ed. B. Sundelius, University of Chalmers, p. 341

Vavrek, R. 2001, PhD Dissertation, Paris Observatory

Vázquez-Semadeni, E. 1994, ApJ, 423, 681

Vázquez-Semadeni, E., Ballesteros-Paredes, J., Rodriguez, L.F. 1997, ApJ, 474, 291

Vázquez-Semadeni, E., Gazol, A., \& Scalo, J. 2000, ApJ, 540, 271

von Weizsacker, C.F. 1951, ApJ, 114, 165

Voss, R. 1988, in The Science of Fractal Images, eds. H.O. Peitgen, \& D. Saupe, New York: Springer

Wada, K., \& Norman, C. A. 1999, ApJ, 516, L13

Wada, K., \& Norman, C. A. 2001, ApJ, 547, 172

Westpfahl, D.J., Coleman, P.H., Alexander, J., Tongue, T. 1999, AJ, 117, 868

Williams, J.P., de Geus, E.J. \& Blitz 1994, ApJ, 428, 693

Williams, J.P., Blitz, L. \& Stark, A. 1995, ApJ, 451, 252

Whitmore, B.C., \& Schweizer, F. 1995, AJ, 109, 960

Zielinsky, M. \& Stützki, J. 1999, A\&A, 347, 630

Zhang, Q., \& Fall, S.M. 1999, ApJ, 527, L81 

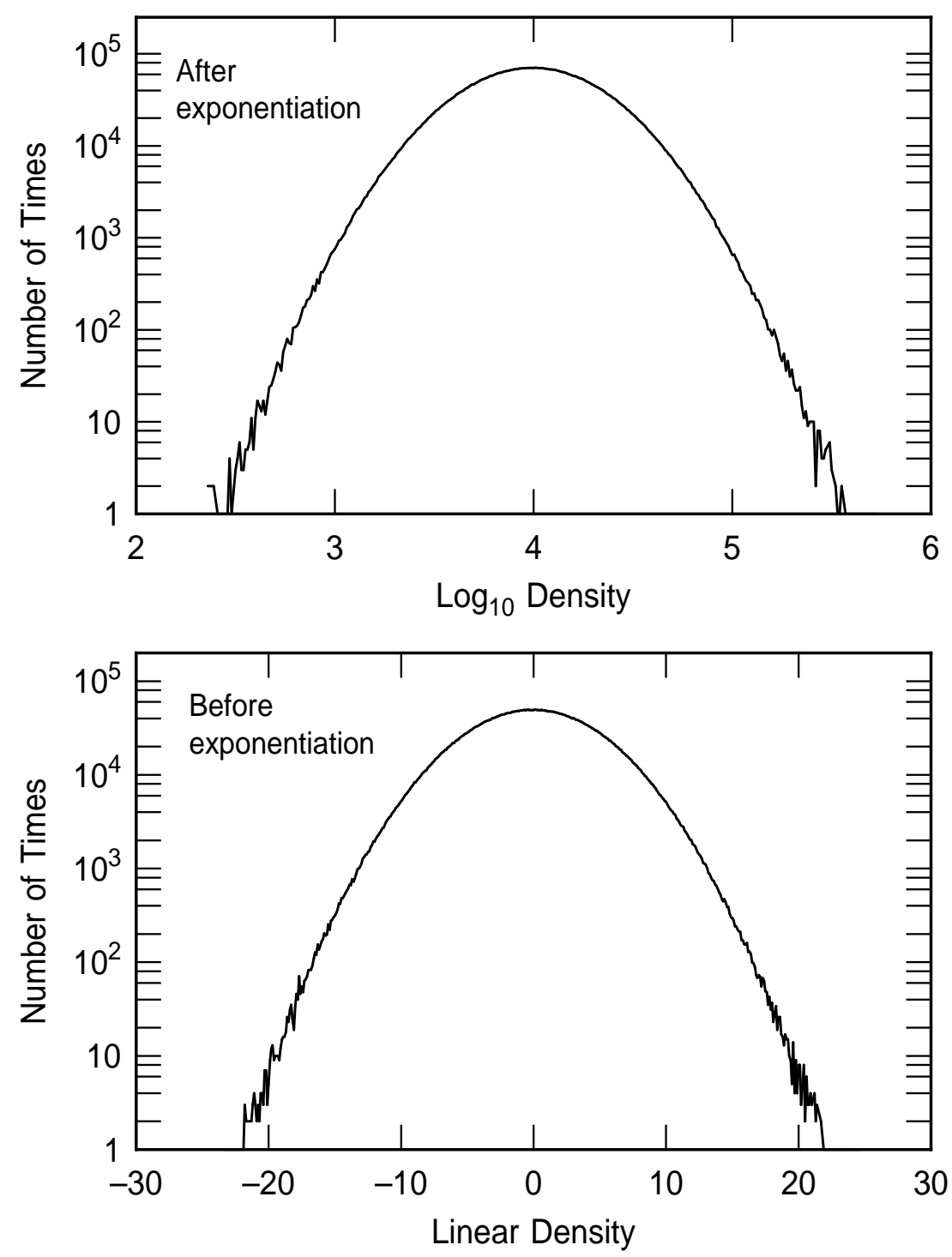

Fig. 1.- (bottom) Histogram of density in the fractal model that was made from the inverse Fourier transform of noise with a power law cut off. (top) Histogram of the fractal model used for the cloud analysis, made from the exponential of the original fractal in order to give a log-normal density distribution function. 


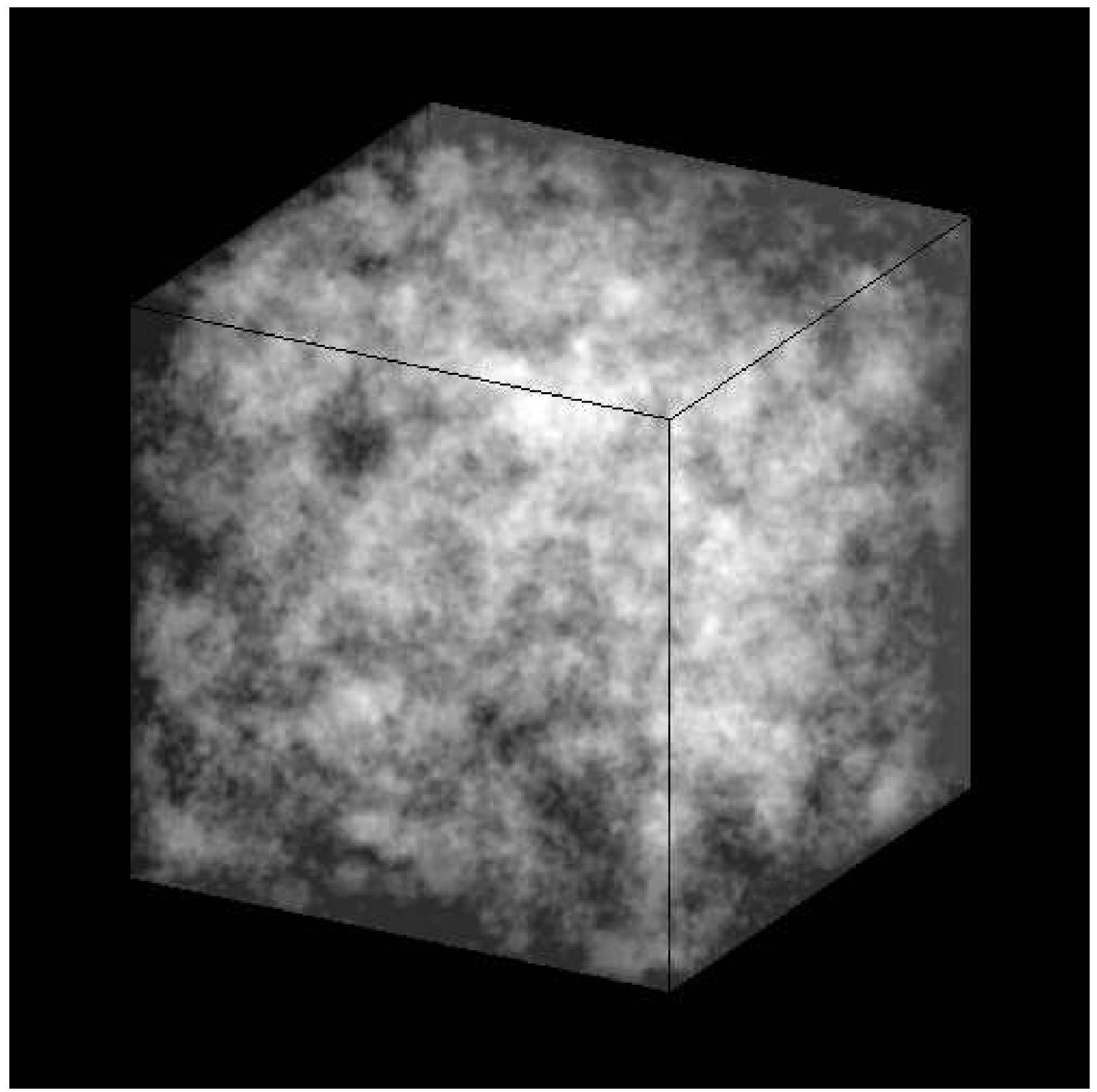

Fig. 2.- Three-dimensional fractal model clipped at 0.03 times the peak intensity. This type of model has the closest agreement to interstellar gas, giving the observed volume filling factor and mass spectrum for clumps. 


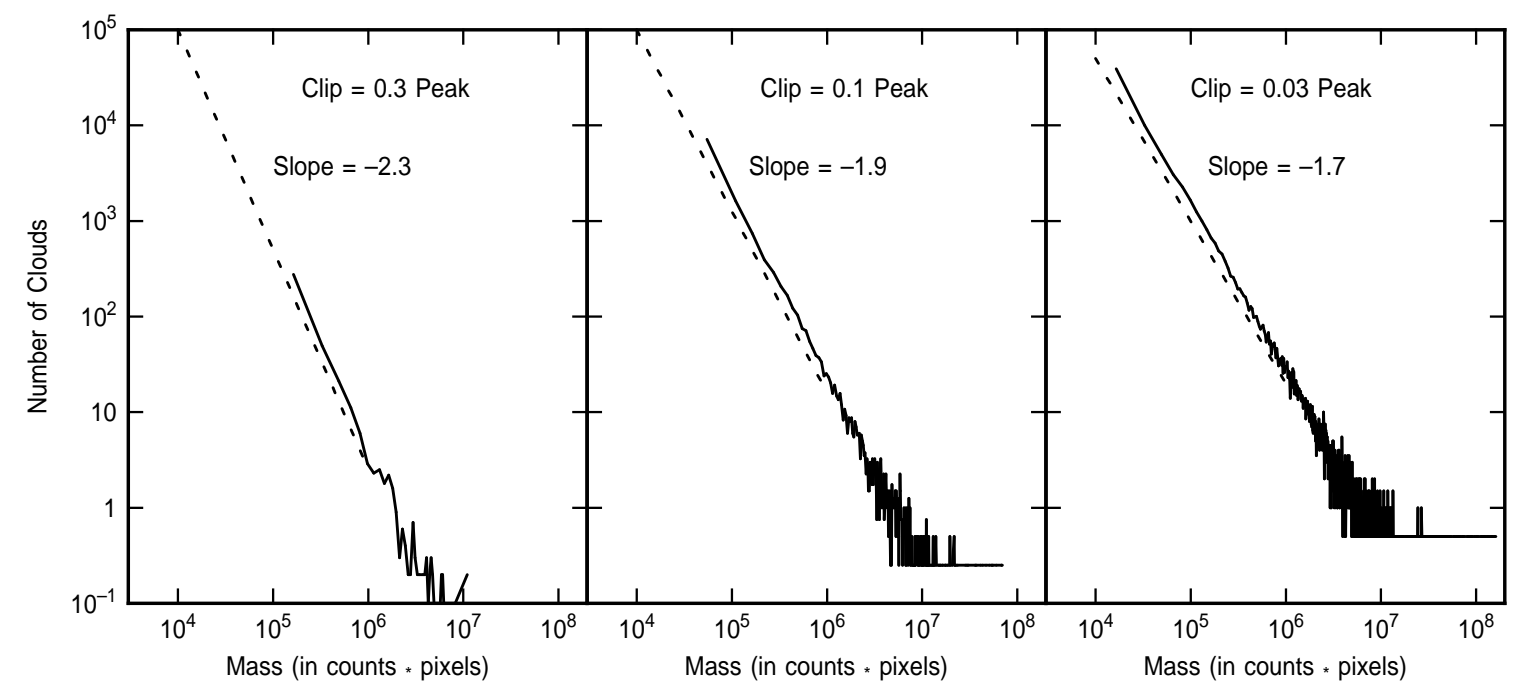

Fig. 3.- Cloud mass spectra for three clipping levels relative to the peak intensity. These spectra are averages over 10, 4, and 2 random model fractals, respectively, and so the minimum counts of $0.1,0.25$, and 0.5 correspond to mass intervals in which only one model had a cloud. The solid curves are the mass spectra and the dashed lines are indicative of the slopes. Lower clipping levels have lower minimum masses and higher maximum masses, but about the same number of clouds at a given mass. The mass is from the sum of the density values in each cloud and is in arbitrary units. 


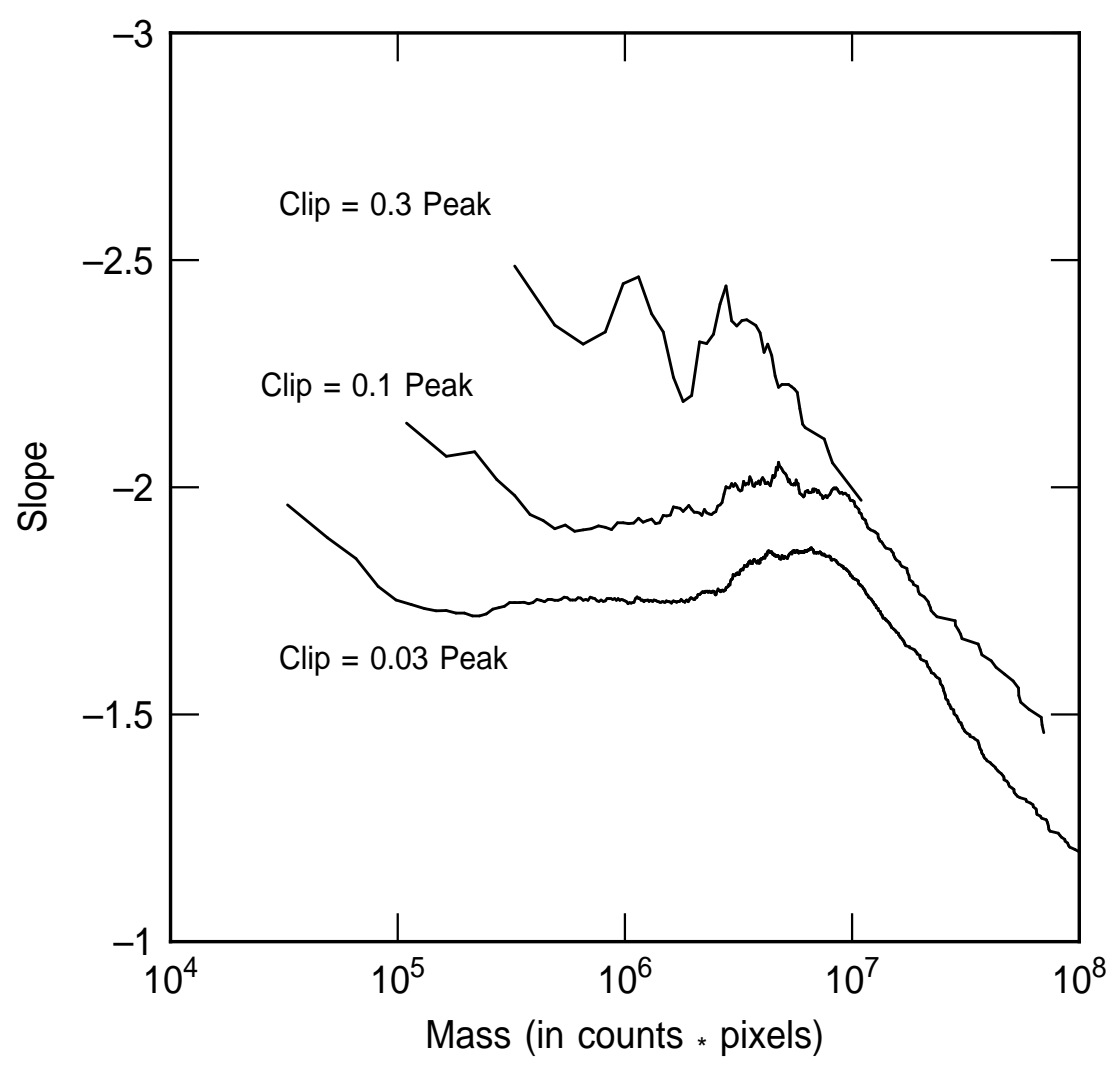

Fig. 4.- Running average slopes of the cloud mass spectra, averaged from the lowest mass up to the mass plotted on the abscissa (in arbitrary units). Results for the three clipping levels are shown. The lowest clipping level gives a power-law mass spectrum with the shallowest slope, $\sim-1.7$. This shallow slope is similar to the observed slope for interstellar clouds and clumps, and it is also from the clipping level that gives the same filling factor as real clouds. The steeper slopes for higher clipping levels might be more appropriate for star clusters and mm-continuum sources, which sample denser regions of clouds. 


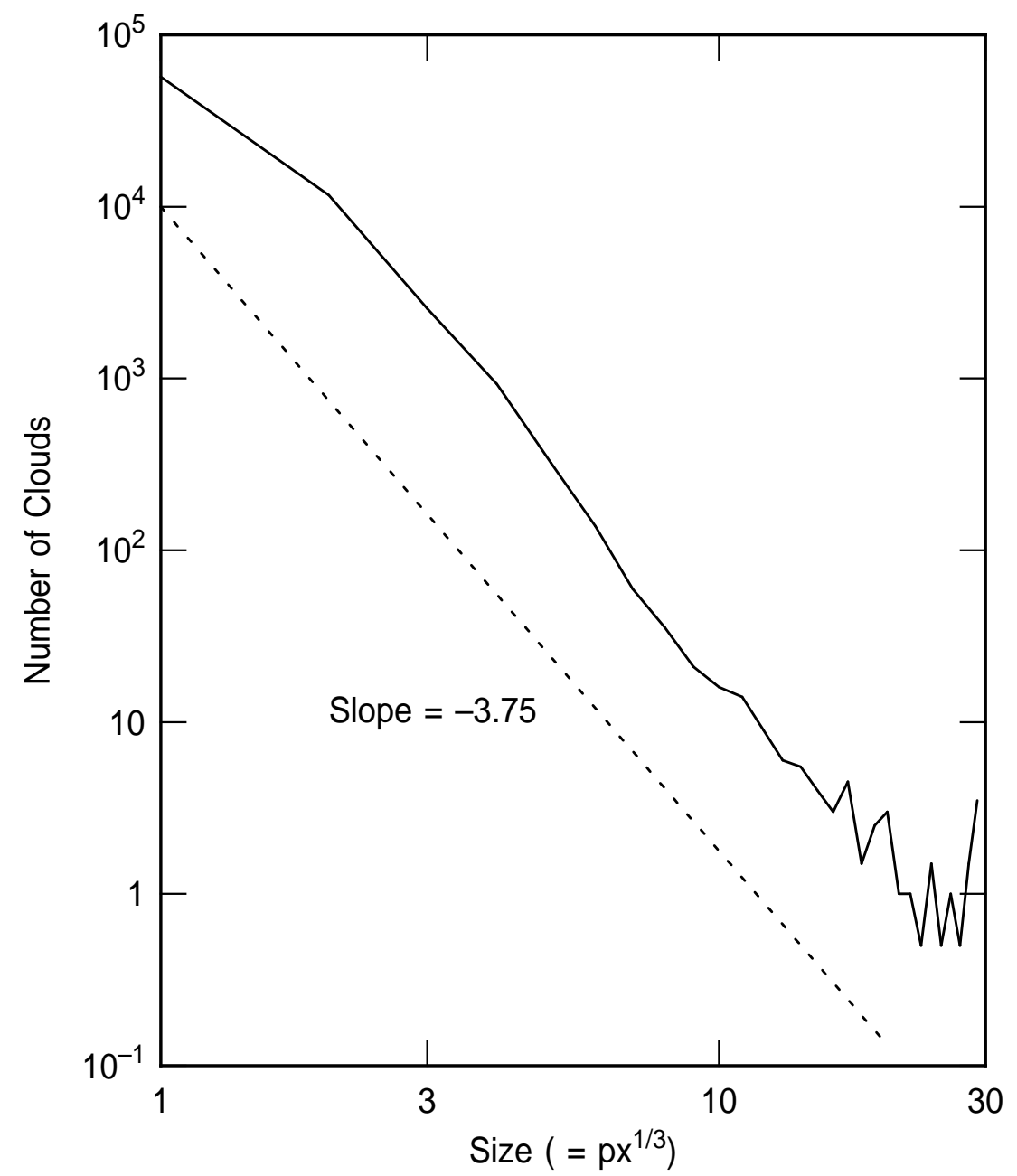

Fig. 5.- Size spectrum for clouds in the model clipped at 0.03 times the peak. The power law nature of the cloud size spectrum leads to the power law mass spectrum even though the density distribution function is not a power law, but a log-normal. The slope of the size spectrum can be used to estimate the average fractal dimension. 


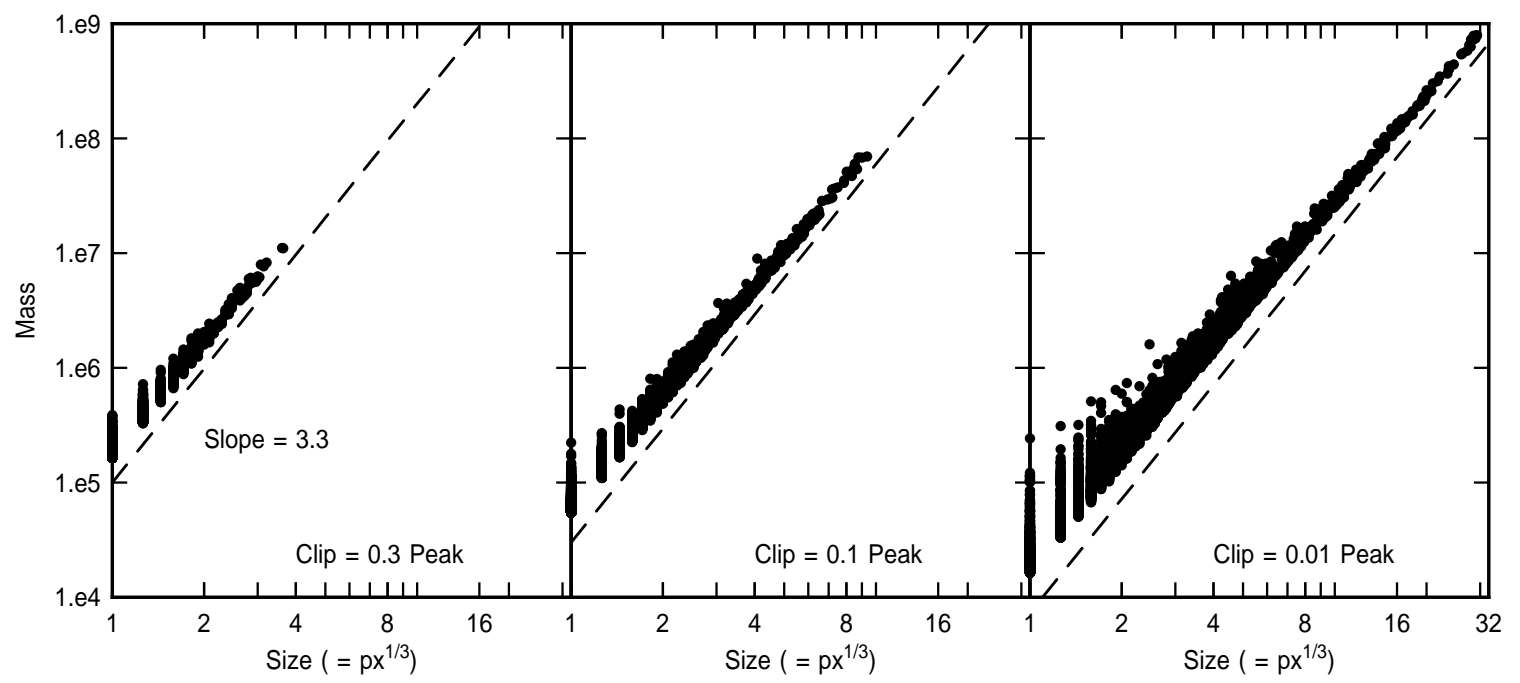

Fig. 6. - Mass versus size for clouds found in the fractal models. The clouds have a nearly constant density that scales with the clipping value used to define them. 


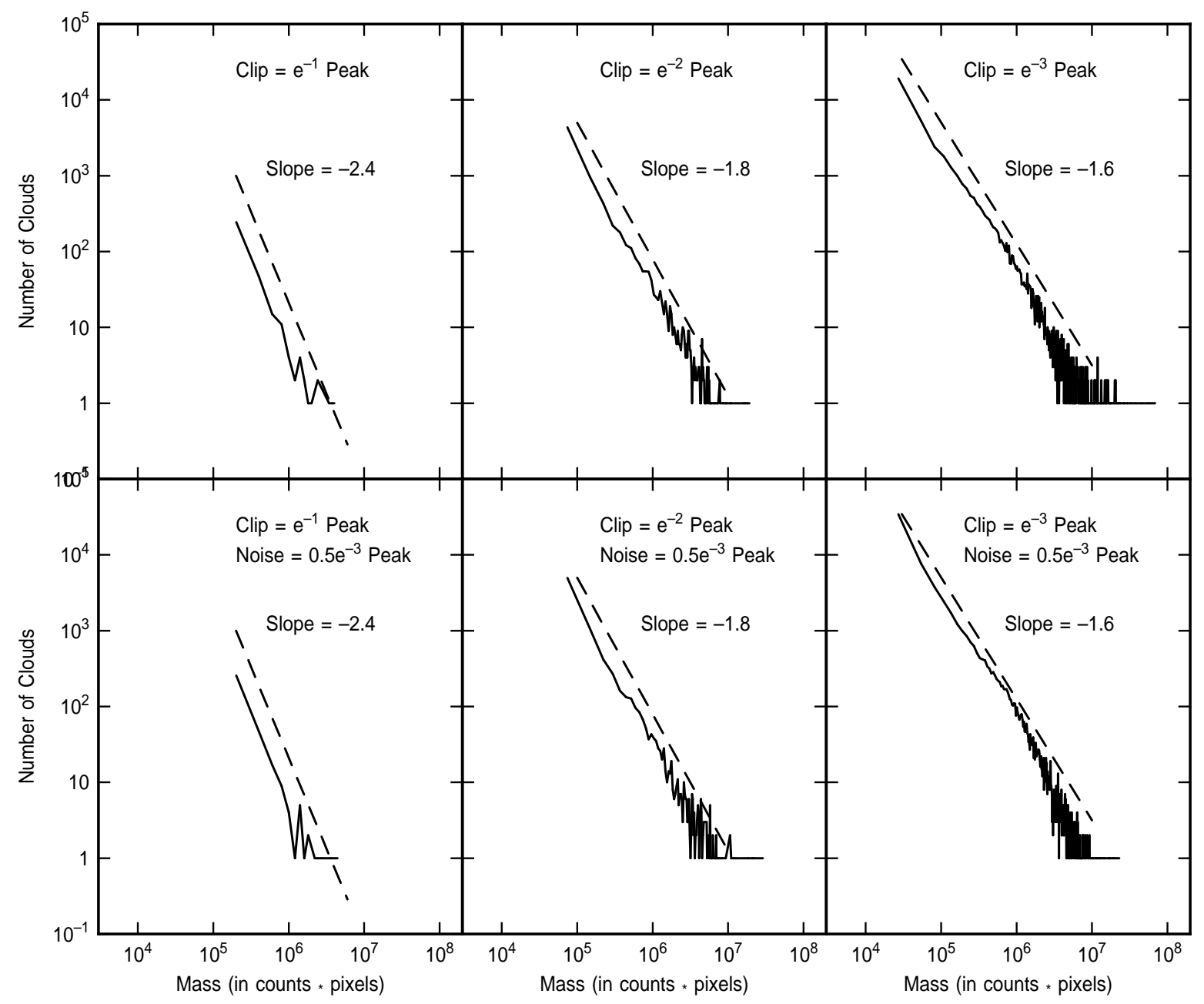

Fig. 7.- Cloud mass spectra for three clipping levels relative to the peak intensity, as in Fig. 3 , but now with clouds defined by the resolved peaks, rather than by all of the connected emission above the clipping level. The top panels are without noise, the bottom panels are for a fractal with noise added at the level of $\pm 0.5 e^{-3}$ times the peak density. Only single models are shown here, not averages of several models as in Fig. 3. The mass is in arbitrary units. 


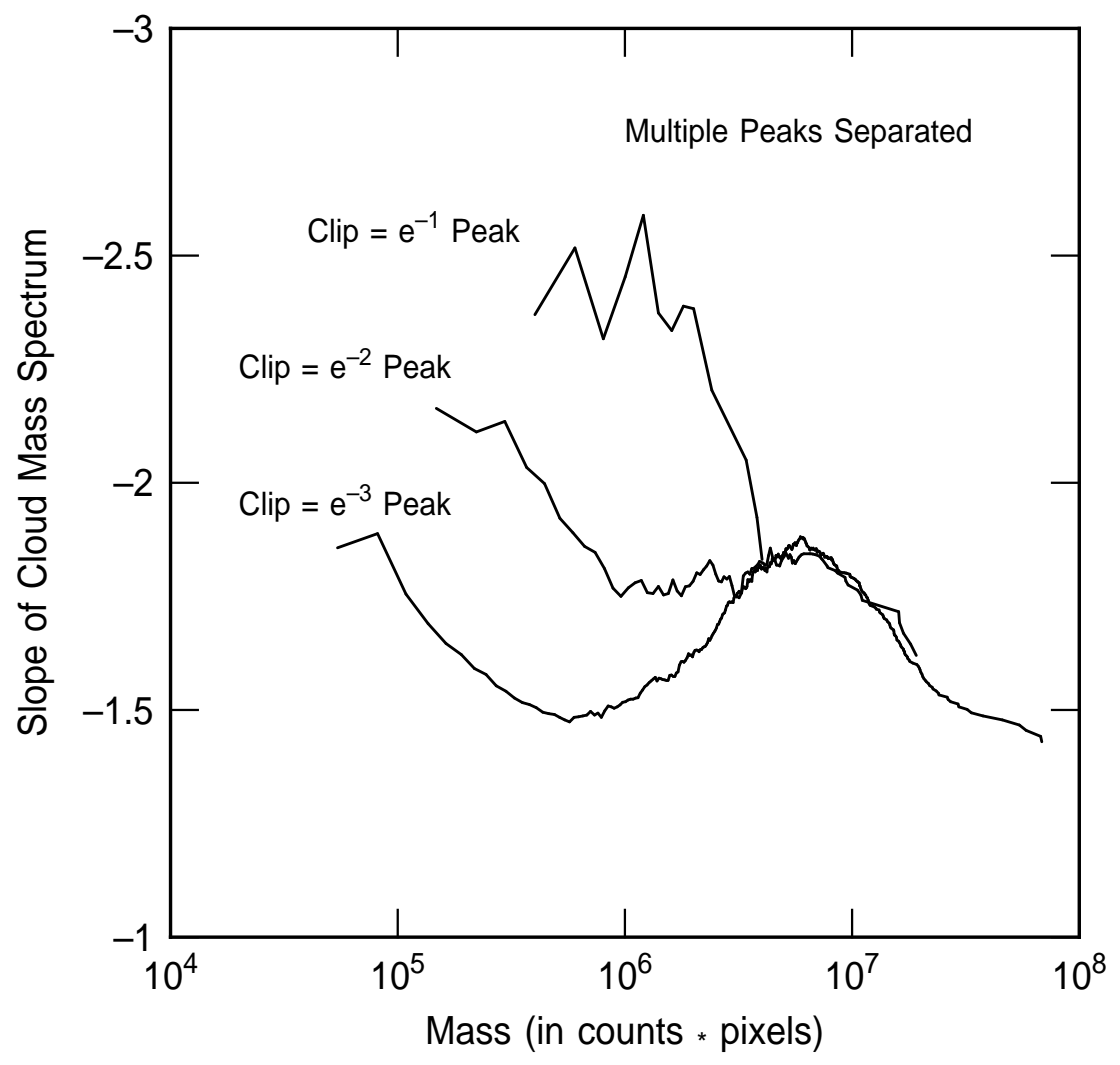

Fig. 8.- Running average slopes of the noise-free cloud mass spectra shown at the top of Fig. 7, averaged from the lowest mass up to the mass plotted on the abscissa (in arbitrary units). Results for the three clipping levels are shown, as in Fig. 4, but now with clouds defined by the resolved peaks. 